mansasa

glyndwhr

Glyndŵr University

Glyndŵr University Research Online

Electrical Engineering

Engineering

9-1-2010

\title{
Rapid Control Prototyping Platform for the Design of Control Systems for Automotive Electromechanical Actuators
}

Roustiam Chakirov

Yuriy Vagapov

Glyndwr University, y.vagapov@glyndwr.ac.uk

Follow this and additional works at: http://epubs.glyndwr.ac.uk/elec_eng

Part of the Electrical and Electronics Commons

Copyright (C) 2010 International Federation of Automatic Control and author. All rights reserved. This is the author's final version of the work after peer review. The article was presented at the $\mathbf{5 t h}$

IFAC Symposium on Mechatronic Systems, held at Marriott Boston Cambridge, USA 13-15 September 2010 and published in the proceedings of Rapid Control Prototyping Platform for the Design of Control Systems for Automotive Electromechanical Actuators Mechatronic Systems in 2010 by Elsevier. The full published article can be found at http://dx.doi.org/10.3182/ 20100913-3-US-2015.00026 on the publisher's website http://www.ifac-papersonline.net/ Detailed/45483.html

\section{Recommended Citation}

Chakirov, R., Vagapov, Y. 'Rapid Control Prototyping Platform for the Design of Control Systems for Automotive Electromechanical Actuators'. [Paper presented at the 5th International Federation of Automatic Control Symposium on Mechatronic Systems, held at Marriott Boston Cambridge 13-15 September 2010], USA Later published in the proceedings of Rapid Control Prototyping Platform for the Design of Control Systems for Automotive Electromechanical Actuators Mechatronic Systems, Volume \# | Part\# 


\title{
Rapid Control Prototyping Platform for the Design of Control Systems for Automotive Electromechanical Actuators
}

\author{
Roustiam Chakirov*, Yuriy Vagapov** \\ * Department of Electrical and Mechanical Engineering and Technical Journalism \\ Bonn-Rhein-Sieg University of Applied Science, \\ 20 Grantham-Allee, Sankt Augustin, D-53757, Germany \\ (roustiam.chakirov@fh-bonn-rhein-sieg.de) \\ ** School of Science and Technology, Glyndwr University, \\ Plas Coch, Mold Road, Wrexham, LL11 2AW, UK \\ (e-mail:y.vagapov@glyndwr.ac.uk)
}

\begin{abstract}
This article introduces and discusses the development of a low-cost Rapid Control Prototyping Platform (RCPP). The aim of RCPP is to automate design of control algorithm of electromechanical actuators and simultaneous implementation it into a target microprocessor. The RCPP is stand-alone system containing software tools and electronic hardware in order to provide all development steps from system identification, model-based control design and code generation up to hardware implementation. The system can be used for development of a torque, speed or position controller for low power electromechanical actuators especially in the area of automotive application. The hardware of the platform is based on a 16-bit microcontroller and includes essential power semiconductor switches, sensors and communication interfaces. The presented RCPP system supports Real-Time-Work interface of MATLAB/Simulink and Calibration Protocol for CAN-Bus communication.
\end{abstract}

Keywords: Actuators, Rapid Control Prototyping, Control System Synthesis, System Identification, Parameter Estimation.

\section{INTRODUCTION}

The number of electric motors, generators and actuators in automotive, aerospace, marine, industrial, and white goods applications is rapidly increasing during the past decades. In automotive industry auxiliary electric motors are widely employed as actuators in the new automotive application areas, such as electrically assisted power steering, drive-bywire and brake-by-wire etc. The customer demand in safety, comfort, economy, clean environment and quality of driving is another reason of intensive implementation of electromechanical actuators in automotive electrical systems.

A few years ago a simple control for the actuators was often sufficient for effective operation. However, today, to improve performance of the actuators its control systems require implementation of more complicate principles of control. On the other hand the preterm of new products (time to market) including actuator applications becomes shorter. It demands reducing of developing cycles of the products such as electromechanical actuator control systems.

The Rapid Control Prototyping (RCP) is aimed to reduce time of development of complex control systems. RCP is widely used in many industrial areas and helps to improve the cost efficiency of research and development of innovative products. The RCP systems are used by vehicle manufacturers for the predevelopment stages and by industry suppliers during the standard development of actuator components.
During past years RCP is the object to develop for designers of computer based systems. There are number of publications and conference contributions in which different aspects and methods of RCP were analysed and discussed. Using RCP in mechatronic systems as in the main area of control application is discussed by Glesner at al (2002), Deppe at al (2004), Kennel at al (2006), Todcher et al (2005). RCP in automotive applications is considered by Lee et al (2004), Dieterle (2005), Munzenberger (2003), Hilgers and Ament (2007), Hoffmann et al (2006).

\section{RCP PLATFORM}

This article describes a RCP platform (RCPP) which is aimed to optimise the procedure of RCP, increase efficiency of prototyping and reduce control system development cost and time. RCPP contains all tools for a quick and reliable development of control system for electromechanical actuators. The platform includes hardware (microcontroller, drivers, data acquisition, interfaces) and software components (operating surface, system identification, model determination and regulator draught).

RCPP provides automatic execution of system identification and system modelling. During the system identification RCPP identify parameters of both electrical motor and mechanical part of the system. The model determination is based on the data acquisition which used for selection of a suitable plant model with possible non-linearity (friction, etc.). 
System identification and model determination are the basis for the next automated parameter calculation of the torque control loop, speed control loop and position control loop. RCPP developing software is equipped by the user interface which is compatible to MATLAB/Simulink and can provide interaction with other control systems.

Unlike existing RCP systems the proposed RCPP decouples the platform from PC-supported simulation and developing methods. System identification, model determination and regulator draughting are processed in the RCPP hardware completely that reduce development expenses and provide know-how clearance.

\section{SYSTEM ARCHITECTURE OF THE RCP PLATFORM}

The hardware includes processor unit, power electronic, instruments to measure motor current and supply voltage, digital I/Os, A/D and D/A converters. The hardware provides communication via CAN-Bus, Serial Peripheral Interface Bus (SPI), RS232/USB interfaces, Ethernet.

The core of the platform hardware is 16-bits $\mu \mathrm{C}$ Infinion $\mathrm{XC167.} \mathrm{This} \mathrm{microcontroller} \mathrm{is} \mathrm{designed} \mathrm{for} \mathrm{automotive}$ applications and often used as a target processor for engine management and other vehicle systems.

The power electronic circuit consists of four high current PWM controlled half bridges in order to commutate one three-phase ac motor or two brush dc motors or electromagnetic actuators. The power electronic circuit can operate at a maximum current of $30 \mathrm{~A}$ and a maximum voltage of $30 \mathrm{~V}$ with PWM frequency up to $25 \mathrm{kHz}$. Each half bridge contains a current sensor which used for torque/force control. The value of PWM is controlled in accordance of the level of supply voltage. The platform provides 8 digital inputs und 8 digital outputs. Also there are $8 \mathrm{~A} / \mathrm{D}$-channels and $8 \mathrm{D} / \mathrm{A}$ cannels. The system supports two CAN channels, two RS232 (or 1xRS232 + 1xUSB) ports and Ethernet interface.

\section{THE RAPID CONTROL PROTOTYPING PROCEDURE}

\subsection{Motion Parameters of Actuator}

At this stage the characteristics, parameters of mechanic motion and requested dynamic behaviour (bandwidth, setting time, system damping) of actuator need to be determined. Also the system limits (limits of rotation angle, maximum and minimum speeds, limits of the voltage and current control) should be defined. The system limits could be taken from system specification or found from the tests applying the moving commands to actuator via user interface.

\subsection{Structure of Actuator Model}

The aim of this stage is to select a basic structure of plant model which presents an actuator including mechanical part of system. Also it needs to specify non-linear system components, for example return spring in valve actuator or nonlinear mechanical transmission with crank shaft. All specified parameters should be input via dialog in user interface.

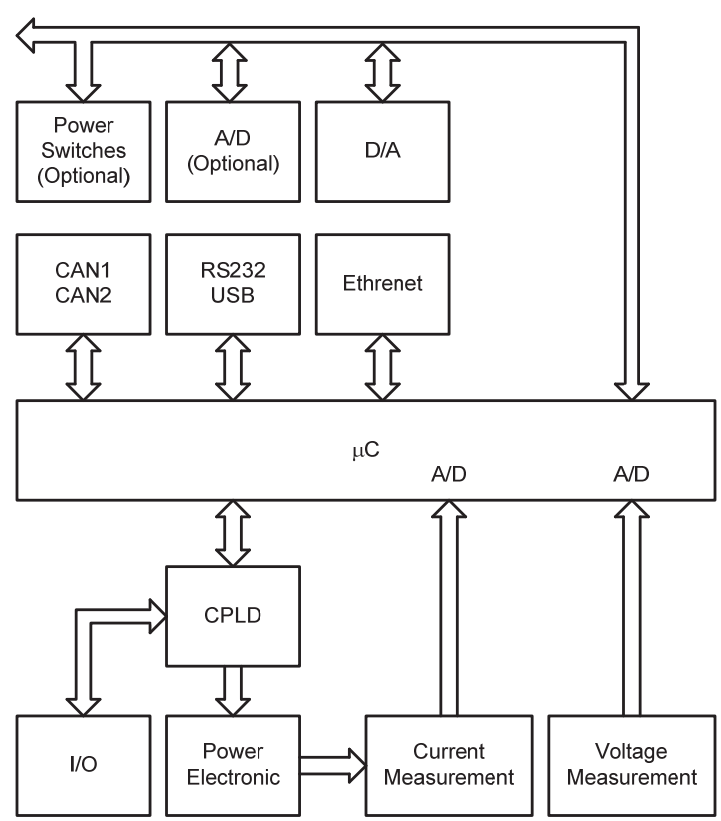

Fig. 1. Hardware block diagram of RCP platform

\subsection{System Identification}

The next step, system identification, is a core procedure of the RCP platform. During this stage the platform generates the test input signals and reads the output signals from the sensors (current, speed, position) using data acquisition. In order to identify the system parameters the input (excitation) signals should be generated as a complex waveform with a rich frequency spectrum. The output signals recorded by data acquisition demonstrate behaviour of the system in control modes: in the torque control mode and in speed control mode with an elementary PI speed controller.

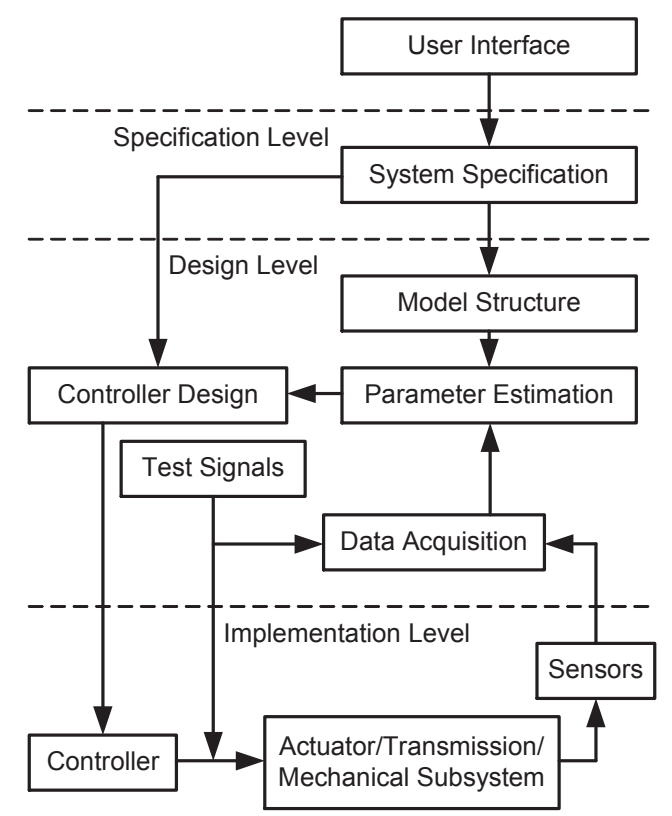

Fig. 2. Block diagram of RCP procedure. 


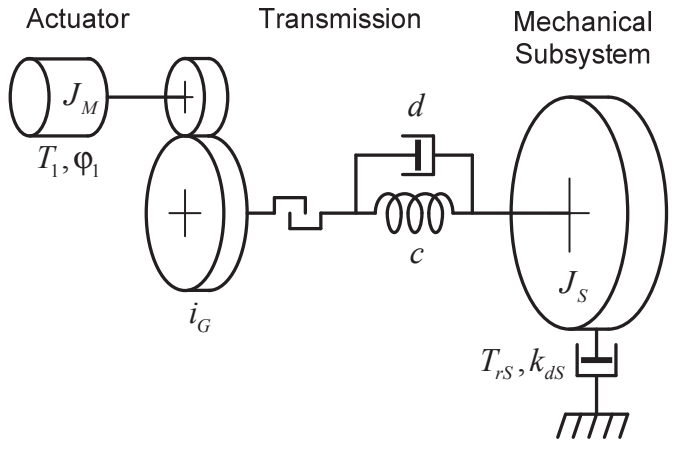

Fig. 3. Schematic diagram of the model.

In order to start the identification procedure the following requirements must be satisfied (Schuette, 2005).

- The current-loop should be represented in the system model as a first order lag element with small time constant. It can be achieved after linearization and optimisation of the current control function.

- The motion limits, torque constant and measurement system characteristics should be known.

\subsection{Plant Parameter Estimation}

The identification method in this step is based on the parametric model assumed by the selection of basic structure for model of plant in the previous steps. The least squares algorithm is applied to estimate the plant parameter using the recorded sampled data (Landau and Zito, 2005).

\subsection{Controller Design and Implementation}

The estimated system parameters are used to build the model based on feed-forward state control. The internal plant model with estimated parameters is generated. Based on the desired regulation behaviour the state-space internal model controller is calculated. The output vector of internal plant model is used for feed-forward control of the plant. The plant state controller is designed to realise dynamic and steady state control system behaviours. The disturbance controller eliminates the parameter estimation errors and disturbances in the system.

\section{PLANT MODEL}

A model complexity depends on selected plant structure. In order to reduce the complexity of simulation model and, as consequence, to deploy a microcontroller a two-inertia structure with torsion spring-dumper link is used as a basic simulation model for mechanical part of system. The schematic diagram of the model is shown in Fig. 3 (Isermann, 2005). The model is based on the assumption that the whole inertia of the system consist of two inertias which are concentrated in two points of the model: actuator side and mechanical side. The inertia of the mechanical transmission is transformed in equivalent inertia to be added to the inertia in the actuator side. The transmission parameters could be presented as a transmission ratio and a mechanical backlash. The transmission ratio must be defined from the system specification. If the transmission ratio is variable or non-linear the static characteristic of the transmission must be defined via user interface. Friction in the model is presented as a combination of Coulomb and viscous frictions.

The state-space model of a plant (actuator and mechanical part) can be described by the following

$$
\left\{\begin{array}{l}
\frac{d \mathbf{x}(t)}{d t}=\mathbf{A} \cdot \mathbf{x}(t)+\mathbf{B} \cdot \mathbf{u}(t) \\
\mathbf{y}(t)=\mathbf{C} \cdot \mathbf{x}(t)
\end{array}\right.
$$

where the vector $\mathbf{x}$ is the state of system defined as

$$
\mathbf{x}^{T}(t)=\left[\begin{array}{llll}
\omega_{A}(t) & \omega_{M}(t) & \varphi_{A}(t) & \varphi_{M}(t)
\end{array}\right]
$$

where $\omega_{A}(t)$ - angular speed of actuator, $\omega_{M}(t)$ - angular speed of mechanical part, $\varphi_{A}(t)$ - angular position of actuator, $\varphi_{M}(t)$ - angular position of mechanical part

The system matrix $\mathbf{A}$ is

$$
\mathbf{A}=\left[\begin{array}{cccc}
-\frac{d_{1}}{J_{A}} & \frac{i_{G} d_{1}}{J_{A}} & -\frac{c_{1}}{J_{A}} & \frac{i_{G} c_{1}}{J_{A}} \\
\frac{i_{G} d_{1}}{J_{M}} & -\frac{i_{G}^{2} d_{1}-\mu_{V}}{J_{M}} & \frac{i_{G} c_{1}}{J_{M}} & -\frac{i_{G}^{2} c_{1}}{J_{M}} \\
\tau & 0 & 1 & 0 \\
0 & \tau & 0 & 1
\end{array}\right]
$$

where $d_{1}$ - damping coefficient, $c_{1}$ - spring constant, $J_{A}$ and $J_{M}$ - moment of inertia of actuator and mechanical part, $i_{G}-$ gear ratio, $\mu_{V}-$ viscose friction number.

The input vector $\mathbf{u}$ is

$$
\mathbf{u}^{T}=\left[\begin{array}{lll}
T_{A} & T_{L} & \operatorname{sign} \omega_{M}
\end{array}\right]
$$

where $T_{A}$ - actuator torque, $T_{L}$ - load torque.

The input matrix $\mathbf{B}$ is

$$
\mathbf{B}=\left[\begin{array}{ccc}
\frac{1}{J_{A}} & 0 & 0 \\
0 & -\frac{1}{J_{M}} & -\frac{1}{J_{M}} T_{C} \\
0 & 0 & 0 \\
0 & 0 & 0
\end{array}\right]
$$

where $T_{C}$ - the torque of dry/coulomb friction.

The system output $\mathbf{y}$ is the angular speed of actuator $\omega_{A}$, which can be found from the system (1).

$$
\mathbf{y}(t)=\mathbf{C} \cdot \mathbf{x}(t)
$$

where $\mathbf{C}=\left[\begin{array}{llll}1 & 0 & 0 & 0\end{array}\right]$. 


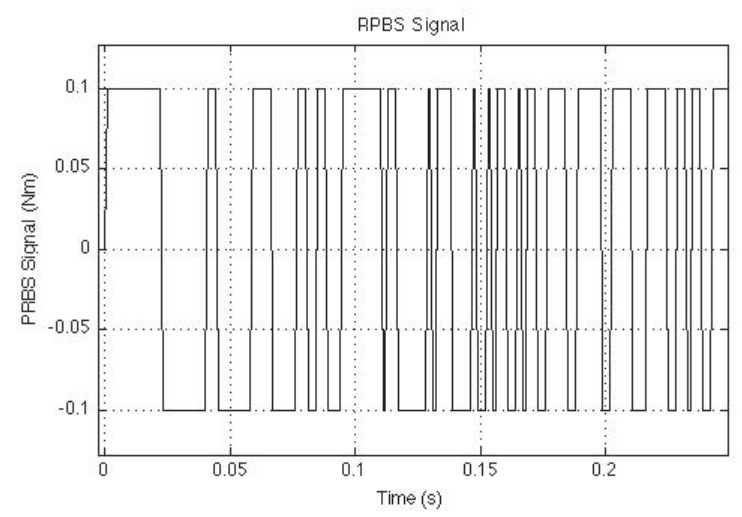

Fig. 4. Pseudo stochastic signal.

The total friction of the system is represented as a torque $T_{F}$

$$
T_{F}=T_{C} \cdot \operatorname{sign} \omega_{M}+\mu_{V} \omega
$$

The dynamic behaviour of a closed-loop actuator torque control is described by a first order equation with small time constant $\tau_{i}$.

$$
G(s)=\frac{1}{\tau_{i} s+1}
$$

The transfer function developed for input torque command $T^{*}$ and output actuator angular speed $\omega_{1}$ is given below.

$$
G(s)=\frac{1}{s \cdot J_{\Sigma}\left(1+\tau_{i} s\right)} \cdot \frac{\frac{J_{M}}{i_{G}^{2}} s^{2}+d_{1} s+c_{1}}{\frac{J_{A} J_{M}}{i_{G}^{2} J_{\Sigma}} s^{2}+d_{1} s+c_{1}}
$$

where $J_{\Sigma}=J_{A}+\frac{J_{M}}{i_{G}^{2}}-$ the inertia of the system referred to the actuator side.

To use the algorithm for parameter estimation the state-space model is transformed into discrete-time polynomial model.

$$
A(q) y(t)=B(q) u(t)
$$

The polynomials $A(q), B(q)$ with unknown parameters contain the time-shift operator $q$. The identification of $A(q)$, $B(q)$ gives possibility to calculate the elements of system matrix $\mathbf{A}$ that leads to determination of system parameters $d_{1}$, $c_{1}, J_{A}, J_{M}, \mu_{V}, T_{C}$.

\section{PARAMETER ESTIMATION}

The parameter estimation method based on measurement and analysis of system frequency response. The data acquisition for frequency response demands a speed control operation of the actuator system. Therefore an elementary PI speed controller based on one mass model should be used.

For parameter estimation of one-mass model with friction the discrete-time function is used as following

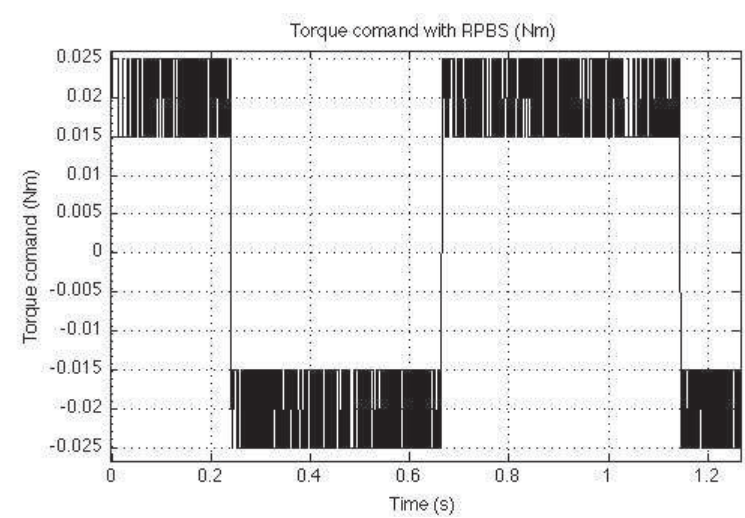

Fig. 5. Torque command with RPBS for on-off control.

$$
\begin{aligned}
& \omega_{A}(k)+a_{1} \cdot \omega_{A}(k-1)= \\
& =b_{1} \cdot\left[T_{A}(k-1)-T_{L}(k-1)-T_{C} \operatorname{sign}\left(\omega_{A}(k-1)\right)\right]
\end{aligned}
$$

where $a_{1}=-e^{-\frac{\mu_{v}}{J_{\Sigma}}}, b_{1}=\frac{1}{\mu_{V}}\left(1-e^{-\frac{\mu_{v}}{J_{\Sigma}}}\right)$.

The vector of unknown parameters $\theta$

$$
\boldsymbol{\theta}=\left[\begin{array}{llll}
a_{1} & b_{1} & b_{1} T_{L} & b_{1} T_{C}
\end{array}\right]^{T}
$$

is estimated from the data measurement vector $\mathbf{m}_{k}$

$$
\mathbf{m}_{k}=\left[\begin{array}{c}
-\omega_{A}(k-1) \\
-T_{A}^{*}(k-1) \\
-1 \\
-\operatorname{sign}\left(\omega_{A}(k-1)\right)
\end{array}\right]
$$

To minimise the prediction error the least-squares method has been used as shown below.

$$
\hat{e}_{k}=\omega_{A}(k)-m_{k}^{T} \cdot \theta
$$

The actuator torque command $T_{A}^{*}$ is a square waveform input combined with a pseudo stochastic signal (RPBS) at a low amplitude (Fig. 4). To generate the actuator torque command $T_{A}^{*}$ a two-step speed controller is used (Fig. 5, Fig. 6).

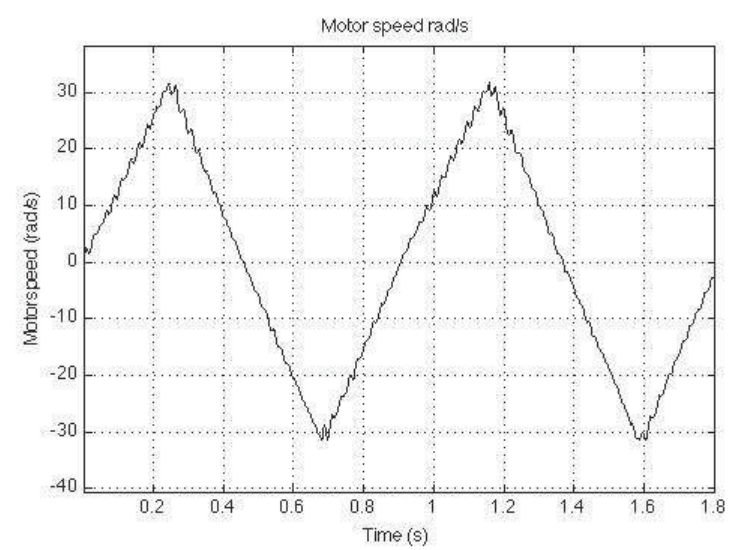

Fig. 6. Motor speed with on-off control 


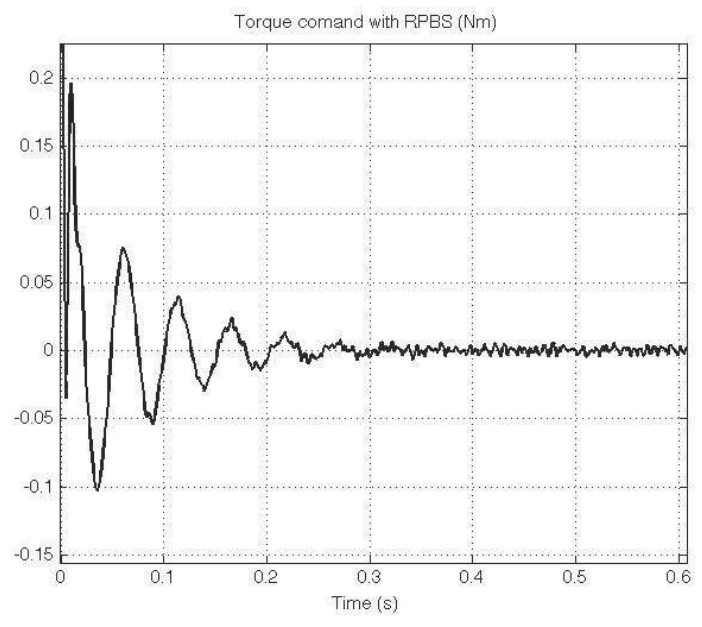

Fig. 7. Torque command with RPBS for PI control

After estimation of the parameter vector $\boldsymbol{\theta}$ the total inertia $J_{\Sigma}$ can be calculated from parameters $a_{1}, b_{1}$.

The parameter of PI controller

$$
G_{P I}(s)=\frac{K_{P}\left(1+s \tau_{N}\right)}{s \tau_{N}}
$$

are calculated using symmetrical optimum $K_{P}$

$$
K_{P}=\frac{J_{\Sigma}}{2 \tau_{i}}
$$

where $\tau_{N}=4 \tau_{i}$.

Using the PI-controller the data acquisition for identification of two-mass system should be performed. The torque command should be superposed with RPBS signal as it is shown in Fig. 7. The required characteristics of PRSB signal for parameter estimation are described by Landau and Zito (2005). The direction of movement (rotation) of the actuator during the measurement has to be the same to avoid the change of Columbian friction and the negative lash influence.

The measurement of input and output data, actuator torque $T^{*}{ }_{A}$ command and actuator speed $\omega_{A}$ are recorded in I/O files over the sampling period (Fig. 8).

The plant transfer function contains a pure integrator. This can be used to reduce the complexity of the model which should be identified. Therefore the input is replaced by its integral while the output remains the same.

$$
u(k)=u(k-1)+T_{A}^{*}(k-1)
$$

To exclude a stationary dc component the mean value of the $\mathrm{I} / \mathrm{O}$ files is calculated and subtracted from $\mathrm{I} / \mathrm{O}$ data. For elimination of a non-stationary dc drift the $\mathrm{I} / \mathrm{O}$ measurements are filtered.

$$
\begin{aligned}
& \omega_{A}^{\prime}(k)=\omega_{A}(k)-\omega_{A}(k-1)+0,2 \omega_{A}^{\prime}(k-1) \\
& u^{\prime}(k)=u(k)-u(k-1)+0,2 u^{\prime}(k-1)
\end{aligned}
$$

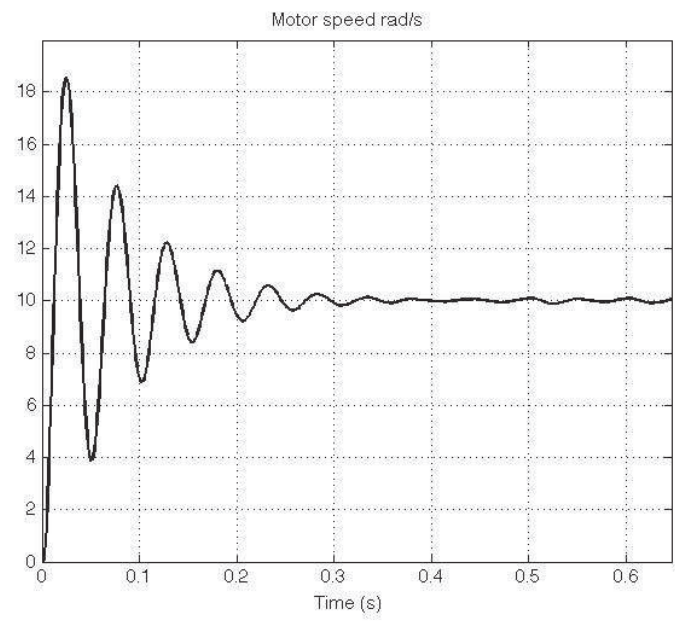

Fig. 8. Motor speed with PI control

The algorithm of parameter identification uses the discretetime model of the plant $G(s)$ without integrator component

$$
\begin{aligned}
& \omega_{A}(k)=-a_{1} \omega_{A}(k-1)-a_{2} \omega_{A}(k-2)- \\
& -a_{3} \omega_{A}(k-3)+b_{1} u(k-1)+b_{2} u(k-2)+b_{3} u(k-3)
\end{aligned}
$$

The model output can be expressed in the form of scalar product between the vector of unknown parameter

$$
\boldsymbol{\theta}^{T}=\left[\begin{array}{llllll}
a_{1} & a_{2} & a_{3} & b_{1} & b_{2} & b_{3}
\end{array}\right]
$$

and the vector of measures

$$
\phi=\left[\begin{array}{c}
-\omega_{A}^{\prime}(k-1) \\
-\omega_{A}^{\prime}(k-2) \\
-\omega_{A}^{\prime}(k-3) \\
u^{\prime}(k-1) \\
u^{\prime}(k-2) \\
u^{\prime}(k-3)
\end{array}\right]
$$

with $\omega_{A}^{\prime}(k)=\boldsymbol{\theta}^{T} \phi(k-1)$.

Parameter estimation algorithm is described by the following recursive least squares estimation equations (Isermann, 2005)

$$
\begin{aligned}
& \hat{\theta}(k+1)=\hat{\theta}(k)+\omega_{A}(k)\left[\omega_{A}(k)-\phi^{T}(k+1) \hat{\theta}(k)\right] \\
& \omega_{A}(k)=\frac{1}{\phi^{T}(k+1) P(k) \phi(k+1)+1} P(k) \phi(k+1) \\
& P(k+1)=\left[\mathrm{I}-\omega_{A}(k) \phi^{T}(k+1) P(k)\right]
\end{aligned}
$$

At initialisation, the parameter vector is set to $\hat{\theta}(0)=0$ and large variances are assumed $P(0)=1000 \cdot \mathrm{I}$. The parameter of the plant transfer function can be calculated after estimation of the vector $\theta$.

Fig. 9 shows the speeds of real and identified systems. 


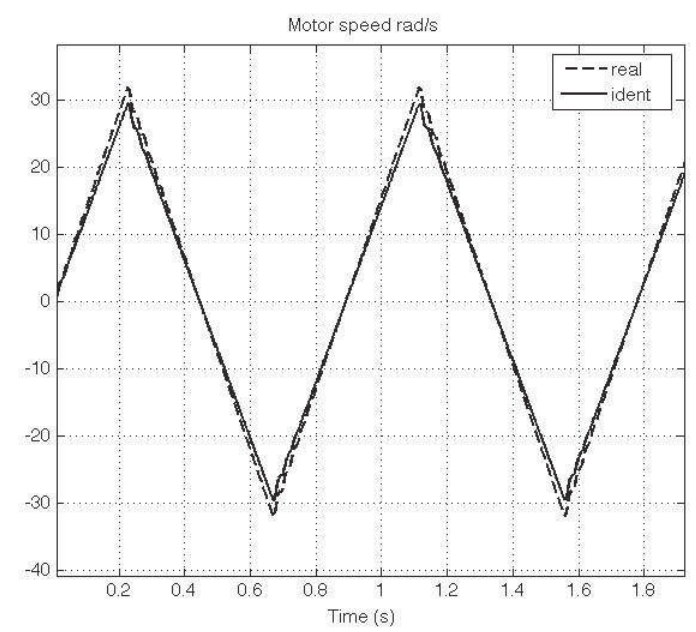

Fig. 9. Comparison of speeds of real and identified systems.

$$
G_{P}(s)=\frac{1}{s \cdot J_{\Sigma}} \cdot \frac{\frac{J_{M}}{i_{G}^{2}} s^{2}+d_{1} s+c_{1}}{\frac{J_{A} J_{M}}{i_{G}^{2} J_{\Sigma}} s^{2}+d_{1} s+c_{1}}
$$

At this stage the coefficients of state variable feedback controller could be determined using pole placement strategy.

The closed loop poles for internal model controller are defined as a third-order polynomial

$$
H(s)=\left(s^{2}+\zeta \omega_{0} s+\omega_{0}^{2}\right)(\tau+s)
$$

The natural frequency of the system $\omega_{0}$ is defined depending on desired setting time and damping ratio.

$$
\omega_{0} \approx \frac{4}{\xi T_{s}}
$$

The damping ratio $\xi$ is chosen to retain the overshoot of less than $5 \%$.

The second-order polynomial determines the dominant poles of $H(s)$. The real pole $-1 / \tau$ will have little effect on the step response if $\tau<<1 / \omega_{0}$.

The state variables from plant model are used as tracking command for plant state controller. The output signal of internal model controller is a feed-forward command.

The plant state controller is designed as internal model controller with reduced dynamics combined PI and state feedback controller. It compensates the error of parameter estimation and achieves zero steady-state error.

\section{CONCLUSION}

The discussed low cost Rapid Control Prototyping Platform based on 16-Bit microcontroller provides quick design and implementation of control algorithms for small electromechanical actuators. The approach of automatic system identification and parameter calculation for speed and position cont- rol gives good results for dynamic systems approximated as two-mass-spring-damper system without distinct nonlinearities and with negligible backlash. The required computing expenditure allows direct integrating of the algorithm in the embedded system based on common industrial 16-bit microcontroller.

\section{REFERENCES}

Glesner, M., Kirschbaum, A., Renner, F.-M., and Voss, B. (2002). State-of-the-art in rapid prototyping for mechatronic systems. Mechatronics, 12, pp. 987-998.

Deppe, M., Zanella, M., Robrecht, M., and Hardt, W. (2004). Rapid prototyping of real-time control laws for complex mechatronic systems: a case study. The Journal of Systems and Software, 70, pp. 263-274.

Kennel, R., El-Kholy, E., Mahmoud, S., El-Refaei, A., and Elkady, F. (2006). Improved direct torque control for induction motor drives with rapid prototyping system. Energy Conversion and Management, 47, pp. 19992010.

Toscher, S., Kasper, R., and Reinemann, T. (2005). Rapid control prototyping of mechatronic systems based on reconfigurable FPGAs. In Proc. Mechatronics 2005: Innovative Product Development, Engineering and Information Technology, Düsseldorf, Germany, pp. 427-448.

Lee, W., Park, S., and Sunwoo, M. (2004). Towards a seamless development process for automotive engine-control system. Control Engineering Practice, 12, pp. 977-986.

Dieterle, W. (2005). Mechatronic systems: Automotive applications and modern design methodologies. Annual Reviews in Control, 29, pp. 273-277.

Munzenberger, R., Dorfel, M., Hofmann, R., and Slomka, F. (2003). A general time model for the specification and design of embedded real-time systems. Microelectronics Journal, 34, pp. 989-1000.

Hilgers, P., and Ament, C. (2007). Control in digital sensoractuator-networks. In Proc. 52nd IWK - Internationales Wissenschaftliche Kolloquium, Ilmenau, Germany, pp. 275-280.

Hoffmann, K., Hesseler, F., and Abel, D. (2006). Rapid control prototyping with Dymola and Matlab for a model predictive control for the air path of a boosted diesel engine. In Proc. New Trends in Engine Control, Simulation and Modelling: E-COSM, Ruel-Malmaison, France, pp. 25-33.

Rettberg, A., Zanella, M., Domer, R., Gerstlauer, A., and Rammig, F. J. Eds. (2007). Embedded System Design: Topics, Techniques, and Trends. International Embedded Systems Symposium (IESS). New York: Springer-Verlag.

Abel, D., and Bollig, A. (2006). Rapid Control Prototyping: Methoden und Anwendungen. Berlin: Springer.

Isermann, R. (2005). Mechatronic Systems - Fundamentals. Berlin: Springer.

Schuette, F. (2002). Automatisierte Reglerinbetriebnahme fur elektrische Antriebe mit schwingungsfähiger Mechanik. Ph.D. dissertation, University Paderborn, Germany.

Landau, I. D., and Zito, G. (2006). Digital Control Systems: Design, Identification and Implementation. New York: Springer-Verlag. 